\title{
SpecSwap-RMC: A Generalized RMC Approach to Structure, Combining Scattering and Spectroscopic Data
}

\author{
Lars G.M. Pettersson
}

\author{
Stockholm University, Stockholm, Sweden; \\ lgm@fysik.su.se
}

Structure determination using Reverse Monte Carlo (RMC) relies on atomistic moves where, after each move, the target property is computed and compared to experiment. The move is accepted if improving the agreement and accepted only with a probability if not. Since of the order a few hundred million moves need to be performed, this requires very rapid evaluation of the property in question. Typical applications include X-ray and neutron scattering and EXAFS in single-scattering mode. Different experimental probes are sensitive to different structural aspects, however, and it can thus be advantageous to combine with, e.g., spectroscopic data to narrow down the range of solutions.

To this end we have developed SpecSwap-RMC [1-3] which performs RMC on a large library of potential structures with precomputed scattering and spectroscopic signals associated with each structure. A subset of structures is selected and all properties built from the selected structures and compared to the experimental data. The RMC is then performed by exchanging structures instead of moving atoms. A set of weights is then generated based on how often each structure is found in the sample set when probed. These SpecSwap-RMC weights can then be used to reweigh the library, to extract an average structure that is consistent simultaneously with all the supplied data.

I will give examples of applications to liquid water, where we combine X-ray diffraction (XRD) and multiple-scattering EXAFS [2], to ice where we use SpecSwap-RMC to analyse what structures have actually been measured in XAS on various samples [4], and discuss XRD, NMR, XAS and XES [5] data on liquid water.

[1] M. Leetmaa, K.T. Wikfeldt, L.G.M. Pettersson, J. Phys.: Cond. Mat. 22 (2010) 135001

[2] K.T. Wikfeldt et al., J. Chem. Phys. 132 (2010) 104513

[3] https://github.com/leetmaa/SpecSwap-RMC.

[4] I. Zhovtobriukh, P. Norman, L.G.M. Pettersson, J. Chem. Phys. 150 (2019) 034501

[5] I. Zhovtobriukh et al., Science China 62 (2019) 107010

Keywords: Reverse Monte Carlo, Spectroscopy, Diffraction 